\title{
Biodegradable Plastic
}

\section{Ashish Chauhan*, Priyanka Chauhan and Bharti Mittu}

Scientist, National Institute of Pharmaceutical Education and Research (NIPER), India

There has been tremendous research going on throughout the world on biodegradable plastics. Biodegradable plastic materials are broadly classified into two main class namely, biodegradable polymers and biopolymers. Biodegradable polymers are the synthetic oil-based polymers that have inherent constituents like poly caprolactone, poly hydroxybutyrate and poly (vinyl alcohol) to ease degradation or are chemically modified to assist biodegradation and are eco-friendly. Biopolymers are naturally occurring polymers such as cellulose, polysaccharides and proteins or derived from these natural polymers. Unlike synthetic polymers these biopolymers are biodegradable moieties and hence they can be decomposed by fungal or bacterial activity into natural metabolites. These biodegradable plastics have a vast application as carriage, packaging material. Most commercially available biodegradable plastic packaging materials are based on natural materials e.g. polysaccharides (cellulose, starch). This is because starch is a renewable, abundant and inexpensive resource. When used alone in packaging applications, starch exhibits a poor performance because of its brittleness and hydrophilic nature. To overcome such problems, starch is often modified mechanically, physically or chemically by combining with a plasticizer or polymeric additives. Where starch is blended with biodegradable polymers or copolymers, the concentration of starch in the mixture is used to classify, label or demarcate the material. The design of biodegradable polymers must ensure its functionality during use, as it may get destructed in response to an environmental effects such as temperature, light, hydration or microbial effect its use. Alternatively, degradation may be triggered by additives that catalyze the breakdown of the polymer chains under specific environmental conditions. Polymers must remain stable during manufacture and use but, they may undergo breakdown rapidly when discarded into landfills. Degradation can be monitored using physical changes or chemical changes, for example, by the observation of new functional groups in Fourier Transform Infrared Spectroscopy (FTIR) spectra. The routine approach to detect degradation is to measure weight loss. But now more sophisticated methods are included for measuring the reduction in molar mass using Gel Permeation Chromatography (GPC) or to determine the loss of tensile properties using an Instron tensile test machine designed to detect changes in mechanical properties. The new materials should be characterized by new techniques and screened before to measure degradation rates including Differential Scanning Calorimetry (DSC), Differential Thermal Analysis (DTA), Thermo Gravimetric Analysis (TGA), Scanning Electron Microscopy (SEM), Chemiluminescence's (CL), Gas Chromatography (GC) and Liquid Chromatography (LC) together with Mass Spectroscopy (GCMS, GCMS-MS, MALDI). The degradation mechanism of biodegradable polymers in an aerobic composting environment is similar to that for organic matter. Biodegradable polymers are attacked and disintegrated by enzymes from naturally occurring microorganisms, such as bacteria and fungi, which are traced under specific conditions in composts. Biodegradation occurs when microorganisms colonize at the surface of the polymer and secrete certain enzymes that break down the macromolecules into fragments. The biodegradation process depends initially upon several factors such as microbial activity, the surface area of the polymer, temperature, $\mathrm{pH}$, molecular weight and polymer crystallinity. The rate of biodegradation is ultimately affected by the environment where the polymer is incorporated, the microorganisms utilized and the nature of the polymeric substrate. In an aerobic composting environment, biodegradable polymers are expected to release carbon dioxide and water. The degradation products must not be toxic or persistent within the environment. Composting of biodegradable polymers is considered as a effective recovery option under the Producer Responsibility Regulations for Packaging Waste. Home composting of biodegradable packaging materials has the potential to divert waste from the municipal waste stream and compliment municipal composting. However, it was noted that polymer residues may be more persistent in compost bins than plant and food waste materials. We hope that upcoming scientist could make the earth free from the treat caused by non degradable polymers, which can have serious consequences if left untreated. *Corresponding author: Ashish Chauhan, Scientist, National Institute
of Pharmaceutical Education and Research (NIPER), India, E-mail:
aashishchauhan26@gmail.com

Received February 04, 2013; Accepted February 05, 2013; Published February 13, 2013

Citation: Chauhan A, Chauhan P, Mittu B (2013) Biodegradable Plastic. J Textile Sci Eng 3: e115. doi:10.4172/2165-8064.1000e115

Copyright: (C) 2013 Chauhan A, et al. This is an open-access article distributed under the terms of the Creative Commons Attribution License, which permits unrestricted use, distribution, and reproduction in any medium, provided the original author and source are credited. 